\title{
Predicting virologically confirmed influenza using school absences in PA
}

\author{
Talia Quandelacy ${ }^{\star 2}$, Shanta Zimmer ${ }^{3,4}$, Chuck Vukotich ${ }^{3}$, Rachael Bieltz $^{3}$, Kyra Grantz $^{1}$, \\ David Galloway ${ }^{3}$, Justin Lessler ${ }^{2}$, Yenlik Zheteyeva ${ }^{5}$, Amra Uzicanin ${ }^{5}$, Hongjiang Gao ${ }^{5}$ \\ and Derek Cummings ${ }^{1,2}$
}

\begin{abstract}
${ }^{1}$ Biology, University of Florida, Gainesville, FL, USA; '2Johns Hopkins Bloomberg School of Public Health, Baltimore, MD, USA; ${ }^{3}$ School of Medicine, University of Pittsburgh, USA, Pittsburgh, PA, USA; ${ }^{4}$ University of Colorado School of Medicine, Department of
\end{abstract} Medicine, Denver, CO, USA; ${ }^{5}$ US Centers for Disease Control, Atlanta, GA, USA

\section{Objective}

To determine if all-cause and cause-specific school absences improve predictions of virologically confirmed influenza in the community.

\section{Introduction}

School-based influenza surveillance has been considered for real-time monitoring of influenza, as children 5-17 years old play an important role in community-level transmission.

\section{Methods}

The Allegheny County Department of Health provided virologically confirmed influenza data collected from all emergency departments and outpatient providers in the county for 2007 and 2011-2016. All-cause school absence rates were collected from nine school districts within Allegheny County for 2010-2015. For a subset of these schools, in addition to all-cause absences, influenza-like illness (ILI)-specific absences were collected using a standard protocol: 10 K-5 schools in one school district (2007-2008), nine K-12 schools in two school districts (2012-2013), and nine K-12 schools from three school districts (2015-2016). We used negative binomial regression to predict weekly county-level influenza cases in Allegheny County, Pennsylvania, during the 2010-2015 influenza seasons. We included the following covariates in candidate models: all-cause school absence rates with different lags (weekly, 1-3 week lags, assessed in separate models using all other covariates) and administrative levels (county, school type, and grade), week and month of the year (assessed in separate models), average weekly temperature, and average weekly relative humidity. Separately, for the three districts for which ILI-specific and all-cause absences were available, we predicted weekly county-level influenza cases using all-cause and ILI-specific absences with all previously stated covariates. We used several crossvalidation approaches to assess models, including leave $20 \%$ of weeks out, leave $20 \%$ of schools out, and leave 52 -weeks out.

\section{Results}

Overall, 2,395,020 all-cause absences were observed in nine school districts. From the subset of schools that collected ILI-specific absences, 14,078 all-cause and 2,617 ILI-related absences were reported. A total of 11,946 virologically confirmed influenza cases were reported in Allegheny County (Figure 1). Inclusion of 1-week lagged absence rates in multivariate models improved model fits and predictions of influenza cases over models using week of year and weekly average temperature (change in $\mathrm{AIC}=-4$ ). Using grade-specific all-cause absences, absences from lower grades explained data best. For example, kindergarten absences explained $22.1 \%$ of model deviance compared to $0.43 \%$ using $12^{\text {th }}$ grade absences in validation. Multivariate models of week-lagged kindergarten absences, week of year, and weekly average temperature had the best fits over other grade-specific multivariate models (change in $\mathrm{AIC}=-6$ comparing $\mathrm{K}$ to $12^{\text {th }}$ grade). The utility of ILI-specific absences compared to total absences is mixed, performing marginally better, adjusting for other covariates, in 2 years, but markedly worse in 1 year. However, these results were based on a small number of observations.

\section{Conclusions}

Our findings suggest models including younger student absences improve predictions of virologically confirmed influenza. We found ILI-specific absences performed similarly to all-cause absences; however, more observations are needed to assess the relative performances of these two datasets.

Performance of models including week-lagged kindergarten absences to predict virologically confirmed influenza in Allegheny County, PA.

\begin{tabular}{|c|c|c|}
\hline Validation & Model & $\mathrm{R}^{\wedge 2}$ \\
\hline Leave $20 \%$ of data out & Absence & $17.8 \%$ \\
\hline Leave $20 \%$ of data out & Temperature and week & $28.4 \%$ \\
\hline Leave $20 \%$ of data out & Absence, temperature and week & $25.5 \%$ \\
\hline Leave 52 weeks out & Absence & $12.6 \%$ \\
\hline Leave 52 weeks out & Temperature and week & $16.0 \%$ \\
\hline Leave 52 wecks out & Absence, temperature and weck & $27.4 \%$ \\
\hline Leave $20 \%$ of schools out & Absence & $11.3 \%$ \\
\hline Leave $20 \%$ of schools out & Temperature and week & $25.2 \%$ \\
\hline Leave $20 \%$ of schools out & Absence, temperature and week & $42.8 \%$ \\
\hline
\end{tabular}

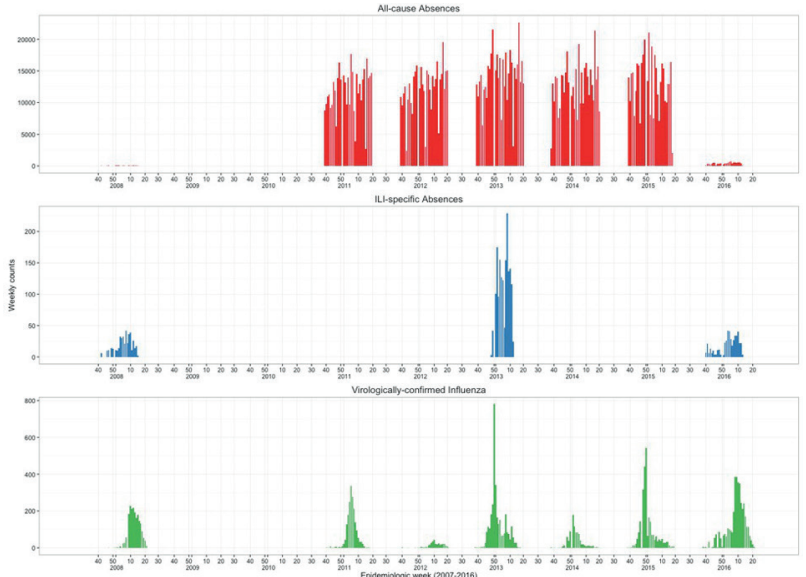

Figure 1. Weekly counts of reported all-cause absences (nine school districts, 2010-2015, a subset of schools in three districts 2007-2008, 2015-2016) (top panel). ILI-specific absences from three school districts (2007-2008, 2012-2013, and 2015-2016) (middle panel) and virologically confirmed influenza all of Allegheny county from 2007 and 2010-2016 (bottom panel).

\section{Keywords}

influenza; surveillance; time; series; school

\section{*Talia Quandelacy}

E-mail: taliaquandelacy@jhu.edu 